\title{
ANSTÄNDIG BLEIBEN \\ Über den Zusammenhang von Respekt und Selbstbewusstsein in unseren Tagen
}

\begin{abstract}
HACKE, A. (2017) Über den Anstand in schwierigen Zeiten und die Frage, wie wir miteinander umgehen, München: Kunstmann, 2017. 192 S., ISBN 978-3-95614-200-0; $€$ 14,95.
\end{abstract}

„Anstand“ und „anständig“ - das sind Wörter, die der Rezensent aus seiner Kindheit in den 60er-Jahren des vergangenen Jahrhunderts kennt und nicht unbedingt in guter Erinnerung behalten hat.

„Jetzt wird es unanständig. . “" hieß es etwa im Elternhaus, wenn in eine Unterhaltungssendung im Fernsehen (Film, Serie oder Show) sexuelle Inhalte eindrangen. „Benehmt euch anständig“ hieß es, wenn die Kinder in den Gottesdienst, zu einer Feier oder sonst wo in die Öffentlichkeit aufbrachen. Die Ermahnung zielte darauf, ja nicht unangenehm (kritisch, ,vorlaut" oder einfach unangepasst) aufzufallen. Mit den Worten „Die haben keinen Anstand“ riss ein Lehrer die Plakate einiger Rockgruppen von den Wänden, mit denen der Klassenraum geschmückt war. Zu häufig wurde noch in der Nachkriegszeit „Anstand“ mit ungefragter Anpassung an Konventionen verwechselt, und folglich geriet der Begriff im großen kulturellen Umbruch der 60er und 70er-Jahre auch kräftig unter die Räder.

Und dennoch greift ein kultur-politischer Essayist unserer Tage auf diesen Begriff zurück, um sich damit auseinanderzusetzen, was in unserer aktuellen Zivilisation schief läuft und wie sich der einzelne dazu verhalten kann. Es handelt sich - und darum sei das Buch den Lesern von EJMH gleich zu Beginn unbedingt ans Herz gelegt -, um einen moral-analytischen Essay mit einem tugend-ethischen Ansatz. Dabei gelingt es dem Autor, vielfältige Alltagsbeispiele aus dem täglichen Leben, aber auch aus der großen Politik mit menschlichen Grundtugenden wie Respekt und Empathie zu verknüpfen - ohne zu moralisieren.

„Über den Anstand in schwierigen Zeiten und die Frage, wie wir miteinander umgehen“ - so lautet der Titel dieses im vergangenen Jahr veröffentlichten Essays, der binnen eines Jahres bereits vier Auflagen erzielte. Das Thema scheint den Nerv der Zeit zu treffen. Axel Hacke ist ein im deutschen Sprachraum ein sehr bekannter Essayist und Kolumnist, der seit vielen Jahren im Magazin der Süddeutschen Zeitung in einer Kolumne das politische Zeitgeschehen kommentiert und in seinen Büchern 
vor allem pädagogische Themen sowie Wert- und Haltungsfragen aufarbeitet. Entlang seines Textes soll es darum gehen,

- was mit dem nicht unumstrittenen Begriff „Anstand“ gemeint sein kann oder muss,

- wofür dieser Anstand wichtig ist,

- was ihn bedroht - vielleicht in unseren Tagen in besonderer Weise -,

- und wie Anstand und Respekt lebenslang gefördert werden können.

\section{Anstand - Annäherungen an eine mehrdeutige Tugend}

Wie bereits eingangs dargestellt, eignet den Begriffen „Anstand“ oder mehr noch „,anständig“ und vor allem dem Gegenbegriff ,unanständig“ eine Ambivalenz. Sie laufen Gefahr, kulturell und gesellschaftlich kontextgebundene Vorstellungen einfach zu verabsolutieren und ,fragwürdige" Normen und Moralen im zwischenmenschlichen Umgang im Allgemeinen und in der Erziehung (,Kinderstube“) im Besonderen unreflektiert hochzuhalten. Dennoch sieht sich Hacke auf diesen Begriff angewiesen, um sich kritisch mit dem aktuellen Umgang miteinander in Politik und Alltag auseinanderzusetzen. „Anstand“ erscheint ihm als Tugend geeignet, ja notwendig, wenn man sich den wachsenden schamlosen oder sogar "lustvollen“" Regelverletzungen in der populistisch gefärbten Politik, in der nur auf den eigenen Vorteil bedachten Wirtschaft, aber auch der Rücksichtslosigkeit unter uns normalen Mitmenschen - z.B. im Straßenverkehr - entgegenstellen will. Das Fass zum Überlaufen bringen die Auswüchse von Beschimpfungen, Demütigungen und Verletzungen in den sozialen oder besser asozialen - Netzwerken wie Facebook und Twitter. Deshalb steht nach der Beurteilung des Autors die Frage, wie wir in diesen Zeiten miteinander umgehen wollen, wieder ganz oben auf der Agenda. Das gilt in besonderem Maße dort, wo Verantwortung für die Bildung und Erziehung junger Menschen oder für die Stärkung des gesellschaftlichen Zusammenhalts übernommen werden soll.

Axel Hacke formuliert die Zusammenfassung seiner Ausführungen folgendermaßen: ,Es geht, wenn es um Anstand geht, um eine grundsätzliche Solidarität mit anderen Menschen, ein Empfinden dafür, dass wir alle das Leben teilen, ein Gefühl, das für die großen und grundsätzlichen Fragen genau so gilt wie für die kleinen, alltäglichen Situationen " (157) - und die Konsequenzen daraus im Umgang miteinander. Dem ist grundsätzlich zuzustimmen, allerdings kann das nicht das Ende der Überlegungen sein. Zum einen erscheint dem Rezensenten aus den oben genannten Gründen der Begriff „Anstand“ zu unpräzise für das, was Hacke hier meint, und zum anderen reicht die verdienstvolle Analyse der schwierigen Situation nicht aus, denn: Wenn wir den Verlust von Anstand und Respekt konstatieren und bedauern, muss die Frage folgen, wie wir diese notwendigen Tugenden wieder fördern können. Zuvor benötigen wir allerdings eine Ahnung, was so viele Zeitgenossen dazu treibt, ihren Anstand zu verlieren, d.h. ihren Mitmenschen Achtung, Solidarität und Mitgefühl zu verweigern. Tugenden wie Anstand und Respekt lassen sich nicht dadurch vermitteln, dass man sie predigt, beschwört oder nur einfordert. Sie müssen miteinander gelebt 
werden, und diejenigen, von denen wir sie erwarten, müssen auch die Chance haben, sie zu realisieren.

\section{Mangel an Anstand - ein sich selbst verstärkendes Phänomen?}

In besonderer Weise ist der Mangel an „Anstand“ oder Rücksichtslosigkeit, die in Hass umschlägt, in der jüngeren Zeit in den (sozialen) Netzwerken aufgefallen. Burcu Gültekin PunSMAN (2018) war „Content-Moderatorin“ im deutschen Team, das im Auftrag des Google-Konzerns Facebook kontrolliert und unerwünschte Inhalte entfernt. Sie beschrieb im SZ-Magazin im Januar 2018 ihre Erfahrungen folgendermaßen: Im Sekundentakt musste sie „Handlungen oder Verhalten, die als anstößig gemeldet wurden [löschen, der. Verf.] - in Bildern, Texten oder in Facebook-Live-Streams: Gewalt, Grausamkeit, Hetze, Sadismus, Mobbing, Selbstverletzungen..."

„Jeder Laie“", so berichtet sie in diesem aufwühlenden Artikel, der auch auf Englisch vorliegt, ,, der gesehen hat, was ich am laufenden Band gesehen habe, muss zu der Überzeugung kommen, dass unsere Gesellschaft krank ist. Ich habe Menschen gesehen, die sich an keinerlei soziale Normen hielten, ich habe eine Welt gesehen, in der es keinen Anstand gibt, keine Rücksicht auf andere... "

„Man wird", so ihre zusammengefasste Erfahrung, ,, als Content Moderator sehr schnell zu einem Experten, was Schimpfwörter und Flüche angeht... Auch Mobbing machte mir Kopfzerbrechen, und das nicht nur bei Teenagern: Zu sehen, wie grausam und gnadenlos Menschen andere Menschen behandeln können, hat mich tief verstört."

Wir können es bei solchen abstoßenden, ja unfassbaren Erfahrungen nicht bewenden lassen. Wenn wir unsere Zivilisation nicht aufgeben wollen, müssen wir verstehen (nicht entschuldigen!), warum solche menschenverachtende Äußerungen zustande kommen können. Fragen wir kundige Zeitgenossen (auch dies tut Hacke in seinem Buch in erhellender Weise, etwa rezipiert er Arbeiten von Yuval Harari (4447) Elisabeth Joh (55ff.) und Georg Franck $78 \mathrm{ff}$.), so weisen sie - neben der Enthemmung durch die Möglichkeit zur anonymen und beziehungslosen Kommunikation in den weltweiten Netzen - auf zwei Erklärungsmöglichkeiten hin:

Die eine hat etwas mit tiefer Verunsicherung vieler Mitmenschen zu tun und ihrem damit verbundenen Gefühl, in dieser Verunsicherung nicht ernst genommen oder sogar verachtet zu werden. Wir leben in Zeiten, so formuliert es Hacke, „die wie gemacht sind für jene, die Veränderung, Offenheit, Ungewissheit schätzen“ (41). Aber es gibt auch die anderen, die Sicherheit brauchen, und die das Gefühl haben, dass mit der Wertschätzung dessen, was sie tun bzw. lebenslang getan haben auch der Respekt verschwindet für das, was bzw. besser wer sie sind - und die sich dafür zu „revanchieren“" versuchen. „Wer also halbwegs jung ist und anpassungsfähig, mehrsprachig und gut ausgebildet, wer keinen Grund hat, die Globalisierung zu fürchten, der sollte sich gut überlegen, ob es besonders anständig ist, sich über jene lustig zu machen, denen der Lauf der Welt im Moment ein bisschen zu rasant ist" (130). Wir 
treffen also auf Wut und Aggression auch deshalb, weil sich Menschen schon lange nicht mehr respektiert fühlen - oder anders formuliert: Der Verlust von Respekt scheint bei weitem nicht immer ein einseitiger Vorgang zu sein. Er hat in vielen Fällen eine nicht glückliche Vorgeschichte des (vermeintlichen) Gesichtsverlusts.

Zum anderen gilt als Triebfeder für die Nutzung von face-book, snap-chat, youtube etc. die Erzeugung von Aufmerksamkeit, von Resonanz und damit verbunden, anscheinend von Bedeutung! Viele unserer Zeitgenossen erleben genau das: Dass ihre Bedeutung für andere im realen Leben schrumpft, aus welchen Gründen auch immer, und ersetzt werden muss durch Resonanz in den sozialen Medien. Dort aber herrscht weniger ein Wettbewerb um die Qualität zwischenmenschlicher Aufmerksamkeit, als ein Wetteifern darum, um jeden Preis aufzufallen, und man fällt vor allem auf mit zumindest neuen, darüber hinaus aber skandal-trächtigen, schockierenden, tabu-verletzenden Posts aller Art.

Wahrgenommen zu werden, geschätzt zu werden, Bedeutung zu haben für andere Menschen - wo dieses Grunderlebnis dauerhaft frustriert wird, resultiert ein aggressives Einfordern solcher Wahrnehmung, ggf. mittels psychischer oder sogar physischer Gewalt. Dafür eignet sich nicht nur der Straßenverkehr oder eine Versammlung von Wutbürgern, sondern in besonderem Maße die Anonymität des weltweiten Netzes.

\section{Was uns den Respekt zurückbringen könnte...}

Nun können wir nicht dauernd von unseren Mitmenschen geliket werden. D.h., neben der Frage, wie wir, die wir uns über fehlenden Anstand beklagen, es selbst mit dem Respekt auch für die, die nicht unbedingt respektabel scheinen, halten, haben wir uns auch damit auseinanderzusetzen, wie wir Menschen (aller Altersstufen, aber bei den Kindern und Jugendlichen haben wir da vielleicht doch die besten Chancen) unterstützen, widerständiger zu werden gegenüber vermeintlichem oder realem Aufmerksamkeitsverlust und sich mit guten Argumenten statt Beschimpfungen zu behaupten. Die Aufgabe lautet, die nachwachsende Generation darin zu bestärken, stärker auf eigene konstruktive Veränderungskräfte zu vertrauen und damit den Kreislauf zwischen (vermeintlich) erfahrender Verachtung und wütend-destruktiver Reaktion unterbrechen zu können. Damit sind wir bei pädagogischen und politischen Grundfragen angelangt, die über den angemessenen Umgang mit den sozialen Medien weit hinausgreifen. Weil der Begriff „Anstand“ immer noch für unreflektiertes Moralisieren zu anfällig erscheint, sollte stattdessen von „Respekt“ als Leitperspektive in der Tugenderziehung - ob in der Pädagogik oder der Erwachsenenbildung und in der Politik die Rede sein. Der aus dem Lateinischen stammenden Begriff „Respekt“ umgreift einen ganzen Korb von Bedeutungen, die wir benötigen, um miteinander nicht nur klarzukommen, sondern aneinander zu wachsen: ,,zurückblicken, berücksichtigen, erwarten/ erhoffen, angehen/ betreffen " sind nur die wichtigsten Übersetzungen. Wenn ich jemandem mit Respekt begegne, habe ich ihn umfassend im Blick, bedenke, was er mitbringt, berücksichtige seine Situation. Ich mache mir bewusst, 
dass er mich etwas angeht und erwarte bzw. erhoffe mir von unserer Begegnung, dass wir beide dabei etwas gewinnen können. Einen solchen Respekt schulden wir nun nicht speziell höher gestellten, älteren, wohlhabenderen, mächtigeren oder sonst ausgezeichneten Menschen, sondern alle einander und grundsätzlich auch dem geringsten der Schwestern und Brüder.

Die Fähigkeit, anderen Menschen Respekt entgegenzubringen, ist allerdings ganz stark mit einer eigenen Selbstwert-Überzeugung und dem von Aaron Antonovsky postulierten „Kohärenzgefühl“" verknüpft (BZGA 2001). Dieses beschreibt nicht nur individuelle psychosoziale Gesundheit, sondern auch die Basis sozialer Resonanzfähigkeit. Im Einzelnen unterteilt Aaron Antonovsky das Kohärenzgefühl in drei wesentliche Erfahrungen, die, wenn sie sich tief in die psychosoziale Matrix eines Menschen eingegraben haben, Stabilität und Ich-Stärke ermöglichen. Das macht wiederum Respekt sich selbst gegenüber und damit auch anderen Menschen möglich. Die Grunderfahrungen lauten: Reize aus der inneren und äußeren Welt sind überwiegend geordnet vorhersagbar und verstehbar. Lebensaufgaben sind Herausforderungen, die Mühe und Engagement lohnen. Und: Die Lebensaufgaben sind grundsätzlich lösbar (KEUPP 1999).

Wer allerdings die gegenwärtige soziale Welt betrachtet, kann Zweifel bekommen an der Verstehbarkeit der Kräfte, die in ihr wirken. Wer zum wiederholten Mal trotz Anstrengung eine Prüfung nicht besteht oder auch bei der fünfzigsten Bewerbung um einen Arbeitsplatz nicht zum Vorstellungsgespräch eingeladen wird, wer daran zweifelt, dass die Rente ihm trotz lebenslanger Arbeit ein auskömmliches Leben im Alter und vor allem bei Hilfebedürftigkeit ermöglicht, fragt sich, wofür seine Anstrengungen sich lohnen sollen - wenn er es überhaupt gelernt hat, sich anzustrengen. Das bedeutet: Es gibt viele Entwicklungen um uns herum, die das Bedürfnis nach Sinnhaftigkeit, Gestaltbarkeit und Teilhabe frustrieren können. Und doch gibt es viele Zeitgenossen, die sich nicht entmutigen lassen, die im Kleinen das Ihre dazu beitragen, dass unser Zusammenleben funktioniert, bei denen die Zuversicht die berechtigten und ebenso wichtigen Zweifel überwiegt. Solche psychische Stärke lässt sich bei anderen nicht herstellen, nicht bei den eigenen Kindern, nicht in der beruflichen Bildungs- und Erziehungsarbeit, aber sie lässt sich provozieren.

Um in dieser Welt „mit Anstand“, also solidarisch und respektvoll zu bestehen, braucht es neben der Selbstwert-Überzeugung Empathie und Mut: Jeder Mensch, der uns begegnet, und das gilt für die in der Erziehung anvertrauten Kinder und Jugendlichen in besonderem Maße, trägt ein Universum an Erfahrungen, kognitiven und emotionalen und nicht zuletzt körpergebundenen, mit sich herum, die sein Erleben und Verhalten manches Mal auf den ersten Blick unzugänglich, schwer verständlich, vielleicht abscheulich erscheinen lassen. Erst die nähere, wohlwollende Beschäftigung mit diesem Menschen, mit dem, was ihn bewegt und umtreibt, macht es möglich, ihn anfanghaft zu verstehen und ihm vor allem zu signalisieren, dass man ihn solcher Verstehensbemühungen für wert empfindet. Damit sind wir auf dem Weg von Toleranz, vielleicht sogar einem gewissen Wohlwollen, zu einem wirklichen Respekt: „Du bist es mir wert, dass ich mich auf dich einlasse, du hast Bedeutung für mich und 
meine Anerkennung - denn mich interessieren deine Päckchen, die du mit dir herumträgst, und auch deine Träume. “ Zu dieser Empathie, zum Interesse und zum Verstehen meines Gegenübers, die wirklichen Respekt wachsen lässt, muss Mut hinzutreten. Vertrauen in einen anderen Menschen, offene, nicht festgelegte Erwartungen, erfordert immer Mut, aber der spezifische Mut in der förderlichen Beziehung besteht darin, einen Menschen letztlich auf seinen Wegen, die er zu Beginn vielleicht nicht einmal selbst kennt, gehen zu lassen (WITTRAHM 2013).

Wir leben in einer unruhigen Zeit, in einer Zeit, in der Respektlosigkeit bis hin zu Hass, Orientierungslosigkeit bis hin zu schwachsinniger Unterhaltung, Minderwertigkeitsgefühle bis hin zum Verlust von Selbstachtung und Scham das Zusammenleben an verschiedenen Orten schwer machen. Wir können dagegen halten, indem wir Menschen, die uns begegnen, besonders jungen Menschen, etwas mitgeben, besser gesagt Erfahrungen mit uns mitgeben, in denen wir respektvoll mit ihnen und miteinander umgehen, weil wir uns bemühen um Verstehen statt um Ausgrenzung und den Mut aufbringen, zu antworten, wo wir angefragt werden. Dann tragen wir einen kleinen Schritt bei zur Beantwortung der Frage von Axel Hacke, wie wir mit einander umgehen sollen in Zeiten, die nicht einfach schlechter sind als früher, sondern anders, und andere Herausforderungen bereithalten, uns zu bewähren. Hacke macht mit seinem Essay darauf aufmerksam, dass jeder Einzelne Mitverantwortung trägt, damit im Umgang miteinander nicht Hass und Verachtung das letzte Wort haben. Viele zivilisierte, das heißt, viele respektvoll und ,anständig“ handelnde und kommunizierende Menschen bilden eine Zivilgesellschaft, und nur eine starke Zivilgesellschaft kann die Basis schaffen dafür, dass Anstand und Respekt weder in der Wirtschaft noch in der Politik und auch nicht in den digitalen Medien verloren gehen.

\section{Referenzen}

BZGA (Bundeszentrale für gesundheitliche Aufklärung), Hrsg. (2001) Was erhält Menschen gesund? Antonovskys Modell der Salutogenese: Diskussionsstand und Stellenwert heruntergeladen am 16. Okt 2018 von https://www.bzga.de/infomaterialien/forschung-und-praxisder-gesundheitsfoerderung/band-06-was-erhaelt-menschen-gesund-antonovskys-modell-dersalutogenese/).

KeupP, H. (1999) 'Subjekt sein heute', Wege zum Menschen 51, 136-52.

Punsmann, B.G. (2018), 'Drei Monate Hölle', Süddeutsche Zeitung Magazin (5 Jan 2018) heruntergeladen am 13. März 2018 von http://sz-magazin.sueddeutsche.de/texte/anzeigen/46817/ Drei-Monate-Hoelle.

Wittrahm, A. (2013) 'Etwas vom Leben, vom Lieben, vom Glauben verstehen: Psychologische Beiträge zur Weiterbildung von Seelsorgern, Pastoralpsychologen, Pädagogen, psychologischen Beratern und Mitarbeitern im Gesundheitswesen', in G. IтTZÉs, Hrsg., Cura mentis - salus populi: Mentálhigiéné a társadalom szolgálatában: Ünnepi kötet Tomcsányi Teodóra 70. születésnapjára: Festschrift für Tomcsányi Teodóra zum 70. Geburtstag (Budapest: Semmelweis Egyetem - Egészségügyi Közszolgálati Kar - Mentálhigiéné Intézet) 201-16. 